\title{
Design of optical path for wide-angle gradient-index antireflection coatings
}

\author{
Minfeng Chen, ${ }^{1,2}$ Hung-chun Chang,,$^{1,3}$ Allan S. P. Chang, ${ }^{2}$ Shawn-Yu Lin, ${ }^{2, \star}$ J.-Q. Xi, ${ }^{2}$ \\ and E. F. Schubert ${ }^{2}$ \\ ${ }^{1}$ Graduate Institute of Electro-Optical Engineering, National Taiwan University, Taipei, Taiwan \\ 2Department of Physics, Applied Physics, and Astronomy, Rensselaer Polytechnic Institute, Troy, \\ New York 12180-3590, USA \\ ${ }^{3}$ Department of Electrical Engineering National Taiwan University, Taipei, Taiwan 106-17 \\ ${ }^{*}$ Corresponding author: sylin@rpi.edu
}

Received 20 December 2006; accepted 7 February 2007;

posted 24 April 2007 (Doc. ID 78254); published 5 September 2007

\begin{abstract}
What we believe to be a new principle is introduced for the design and selection of gradient-index antireflection profiles that are effective over a wide range of incident angles as well as wavelengths at a given physical film thickness. It is shown that at oblique incidence the smoothness of the optical path of incident light inside a gradient-index film has a crucial effect on the overall reflection. Thus the smoothness of variations in refractive angle (rather than that of the index profile itself) needs to be maximized for wide-angle operation. As an example, the performance of Gaussian and Quintic profiles at large incident angles are considered in light of this point of view. (C) 2007 Optical Society of America

OCIS codes: $310.1210,310.1620$.
\end{abstract}

\section{Introduction}

Antireflection coating (ARC) is key to suppression of undesired interfacial Fresnel reflections in optical components and devices. While the well-known singlelayer quarter-wave film can in theory lead to zero reflection at a single wavelength, broadband ARC is often needed for many applications. In practice coating materials with the required refractive index for the quarter-wave antireflective (AR) film may not be available. To address these issues, a multilayer stack of homogeneous thin films has been investigated extensively for over half a century [1], resulting in the development of a rich variety of multilayer thin film schemes [2] and design methodologies [3].

An alternative broadband ARC solution is a layer of inhomogeneous film with gradient-index in which the refractive index varies gradually and monotonically along its thickness from the ambient (usually air) index to the substrate index [4]. Many specific gradientindex profiles including Quintic [5], Gaussian [6],

0003-6935/07/266533-06\$15.00/0

(C) 2007 Optical Society of America
Exponential [7], Exponential-Sine [8], and Klopfenstein [9] have been investigated previously. Compared to multilayer uniform films, gradient-index ARC can be less sensitive to the angle of incidence [10], and is thus desirable for use in applications such as solar cells [11] and light-emitting diodes [12] that require effective ARC over a wide range of incident angles and where the gradient-index can be implemented by techniques such as patterning of subwavelength surfacerelief "moth eye" structures [13]. Yet, while numerous designs of multilayer ARC for oblique incident angles were previously reported [14-18], there is relatively little literature on the design and performance of gradient-index ARC at grazing incident angles. Recently, Poitras and Dobrowolski [19] pointed out that at oblique angles, a smooth variation of the spatially dependent refractive angle inside a gradient-index ARC is necessary to reduce polarization splitting in reflectance of the film. They also noted that performance is significantly degraded at large refractive angles due to deformation of the index profile as seen by the light. To partly remedy this, they showed that by applying a spatial scaling to an index profile that effectively elongates the portion close to the ambient 
side, the resultant modified profile has improved performance at large incident angles over its original counterpart. However, this comes at the expense of increased physical thickness of the film and slight performance degradation at near-normal incidence.

The purpose of this paper is to report, what we believe to be, a new principle for the design and selection of gradient-index ARC profiles that are effective over a wide range of incident angles as well as wavelengths, without the need for an extension of the film's thickness. It is shown that large variations in the optical path (which is characterized by the refractive angle) of incident light inside a gradient-index film directly lead to an increase in the overall reflection at oblique incidence. This effect becomes more prominent at large angles. Thus, it is the smoothness of variations in refractive angle rather than that of the index profile itself that needs to be maximized for wide-angle operation. As an example, we show that the Gaussian profile outperforms the Quintic at large incident angles and consider this result in light of the refractive angle point of view.

\section{Results and Discussion}

\section{A. Design of Optical Path}

The geometry of a gradient-index ARC is shown in Fig. 1. The inhomogeneous film is placed on a substrate with an index $n_{\max }$, and the ambient is assumed to have an index $n_{\min }$. The ambient-film interface is at $z=0$ while the film-substrate interface is at $z=d$, where $z$ is the physical distance normal to the film surface. Inside the film the refractive index $n(z)$ varies continuously from $n_{\min }$ to $n_{\max }$. All media are assumed to be nondispersive and nonabsorbing.

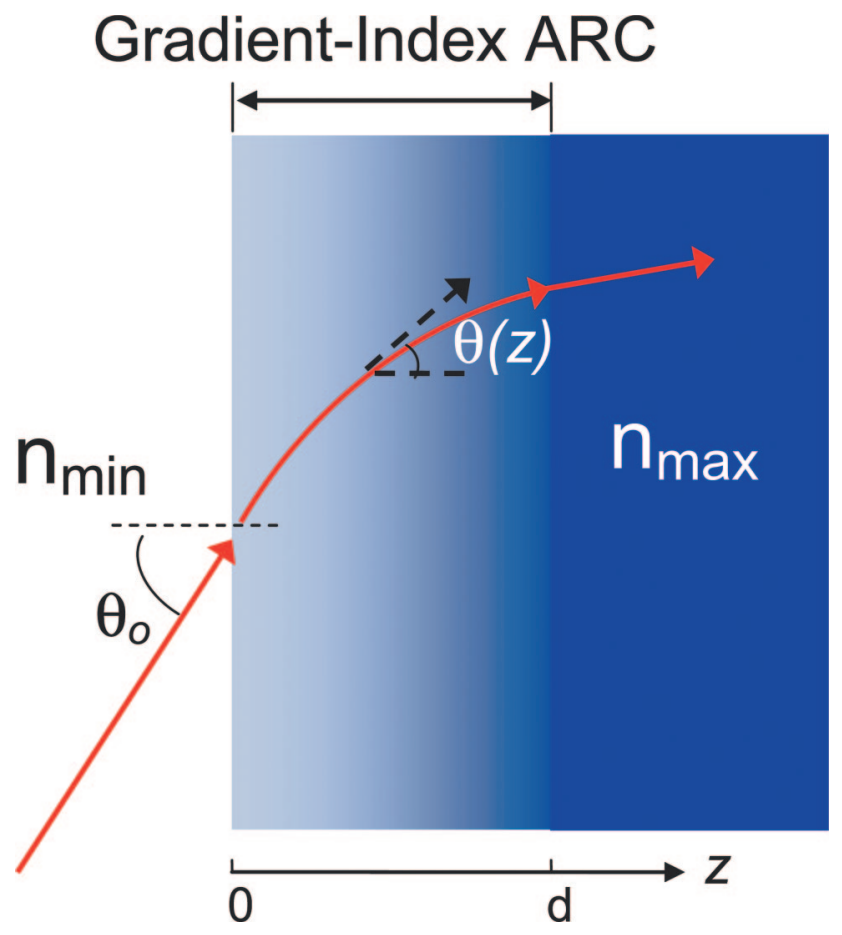

Fig. 1. (Color online) Geometry of a gradient-index ARC.
Light is incident upon the film surface from the ambience at an angle $\theta_{0}$, and inside the film the tangential propagation direction of light is characterized by the spatially dependent refractive angle $\theta(z)$.

For a gradient-index profile, it is important to consider the optical thickness $x$. The incremental optical thickness $\Delta x$ is related to the incremental physical thickness $\Delta z$ by $\Delta z=\Delta x / n(x)$, so that

$$
z=\int_{0}^{x} \frac{d x^{\prime}}{n\left(x^{\prime}\right)} .
$$

At oblique incidence, the optical characteristics of the film are different for TE and TM incident polarizations. We can define the characteristic index for the two polarizations in terms of the optical thickness as

$$
\begin{gathered}
n_{\mathrm{TE}}(x)=n(x) \cos (\theta(x)), \\
n_{\mathrm{TM}}(x)=n(x) / \cos (\theta(x)),
\end{gathered}
$$

where $\cos (\theta(x))$ is obtained by

$$
\cos (\theta(x))=\left[1-\frac{n_{\min }}{n(x)} \sin \left(\theta_{0}\right)\right]^{1 / 2} .
$$

The refractive angle within the coating, $\theta(x)$, can be obtained from Snell's law, that determines the optical path of light propagating inside the coating. By the law of reflection at the interface of two optical materials (Fresnel reflection), the incremental reflectance $\mathrm{d} r_{\mathrm{TE}}(x)$ within the coating for the TE polarization is then given by

$$
\begin{aligned}
\mathrm{d} r_{\mathrm{TE}}(x) & =\left[\frac{n_{\mathrm{TE}}(x)-n_{\mathrm{TE}}(x+\mathrm{d} x)}{n_{\mathrm{TE}}(x)+n_{\mathrm{TE}}(x+\mathrm{d} x)}\right]^{2} \\
& \approx\left[\frac{\mathrm{d} n_{\mathrm{TE}}(x)}{2 n_{\mathrm{TE}}(x)}\right]^{2} .
\end{aligned}
$$

The incremental reflectance for TM polarization has exactly the same form but with $n_{\mathrm{TM}}(x)$ instead. We can treat the continuous index function as an infinite series of discrete lamellar layers of thickness of $d x$ and calculate the reflectance as a function of $x$ directly by the Fresnel equation, Eq. (4). At a large angle of incidence, $\cos (\theta(x))$ will approach zero, leading to a great variation of the characteristic index in tiny increments of $\theta(x)$. Therefore, the reflectance would be significantly increased according to Eq. (4). Based on this argument, we make an assertion: the variation of refractive angle within the coating has great influence on the performance of gradient-index ARC.

It is well known that smooth refractive index profile is needed for optimal ARC. Now we have an alterative point of view: building an index profile in which the refractive angle is smoothly varied within the coating. As an example, we choose a profile 


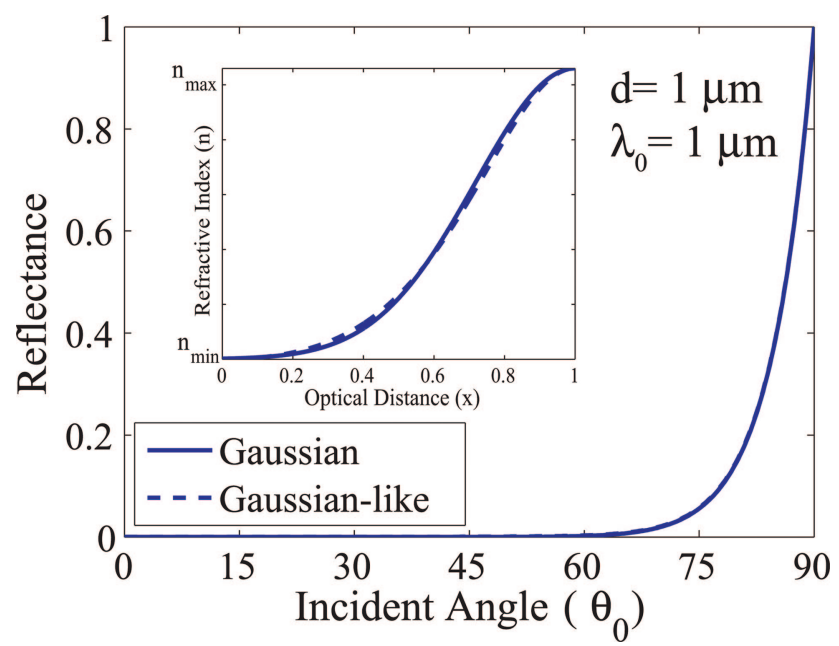

Fig. 2. (Color online) Comparison of reflectance under Gaussian and Gaussian-type profiles for a wavelength of $\lambda=1 \mu \mathrm{m}$ with $d$ $=1 \mu \mathrm{m}$ physical thickness. Inset is the Gaussian and Gaussiantype profile.

wherein the variation of refractive angle is a wellknown smooth sinusoidal function at incident angle of $\theta_{0}=75^{\circ}$ :

$$
n(x)=\frac{n_{\min } \sin \theta_{0}}{\sin \left\{\theta_{0}+\left(\theta_{1}-\theta_{0}\right)[1-\cos (\pi x) / 2]\right\}},
$$

where $\theta_{1}$ is the refractive angle in the substrate. The index profile of Eq. (5) is in fact very close to a Gaussian profile of the form

$$
n(x)=n_{\min }+\left(n_{\max }-n_{\min }\right) \exp \left[-\left(\frac{x-1}{0.4}\right)^{2}\right],
$$

as illustrated in Fig. 2 and shows similar reflectance as the Gaussian profile. We refer to this profile as a Gaussian-type profile.

To illustrate the effect of smoothness of refractive angle profile, we next compare the performance of the Gaussian given by Eq. (6) and Quintic profile of the form

$n(x)=n_{\min }+\left(n_{\max }-n_{\min }\right)\left(10 x^{3}-15 x^{4}+6 x^{5}\right)$.

These two index profiles are expressed in terms of their optical distance, $x$. While the physical thickness is the actual coating thickness, the optical thickness can be regarded as the imaginary thickness seen by the electromagnetic wave. With optical thickness, $x=1 \lambda_{0}$, where $\lambda_{0}$ is the wavelength in vacuum, Quintic exhibits physical thickness of $z_{\text {Quintic }}=0.7 \lambda_{0}$, while Gaussian has $z_{\text {Gaussian }}=0.78 \lambda_{0}$.

Shown in Fig. 3 is the calculated reflectance of the two profiles as a function of incident angle. Insets are the zoom-in diagrams. The light is assumed to be incident from air $\left(n_{\min }=1.0\right)$ with wavelength of $\lambda_{0}=1 \mu \mathrm{m}$ and the substrate is assumed to be aluminum nitride $\left(n_{\max }=2.06\right)$. Two physical thicknesses are considered of $1 \mu \mathrm{m}$ and $3 \mu \mathrm{m}$. In Fig. 3 one can (a)

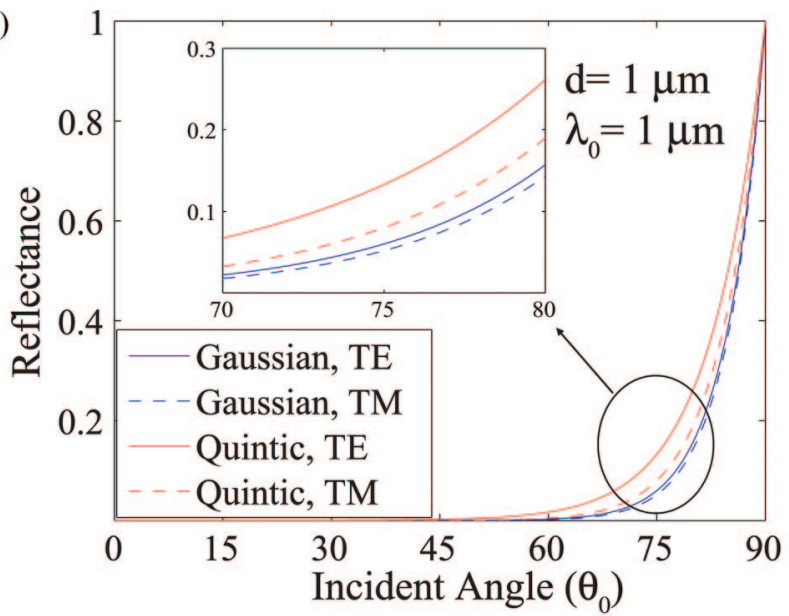

(b)

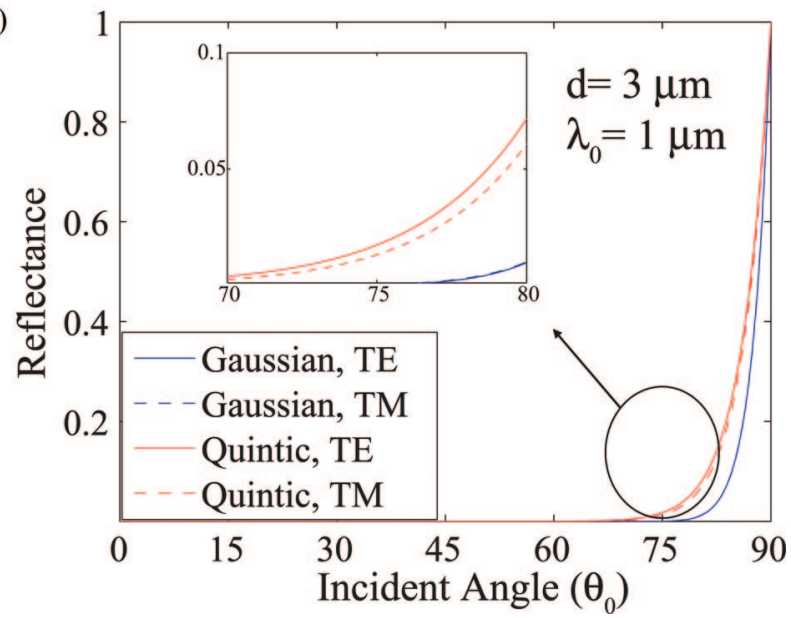

Fig. 3. Gaussian and Quintic gradient-index coating angular reflectance calculated for a wavelength of $1 \mu \mathrm{m}$ with a physical thickness of (a) $1 \mu \mathrm{m}$ and (b) $3 \mu \mathrm{m}$.

see that the Gaussian and Quintic profiles show little difference at low incident angle. However, the significant difference is revealed once the incident angle goes to above $60^{\circ}$. Furthermore, the TE polarization is indiscernible to the TM polarization under Gaussian while the polarizations are split with Quintic. We consider the reduced polarization splitting under the Gaussian profile to be evidence that variation of the refractive angle in Gaussian is smoother than that in the Quintic.

It is obvious that the $3 \mu \mathrm{m}$ coating gives a better performance than the $1 \mu \mathrm{m}$ coating due to the smoother index variation. If an unpolarized light is illuminated from $\theta_{0}=0$ to $\theta_{0}=\pi$ uniformly, we may obtain the average reflectance by integrating the reflectance's overall angle and dividing the integration by $\pi$. For $1 \mu \mathrm{m}$ thickness, the Gaussian and Quintic give an angle-averaged reflectance of $5.93 \%$ and $7.53 \%$, respectively. The result shows that the Gaussian profile reduces reflectance by about $1.6 \%$ compared with Quintic.

Figure 4(a) shows the reflectance with a different wavelength at coating thickness of $1 \mu \mathrm{m}$. The unpolarized reflectance is given by the average of $\mathrm{TE}$ and 
(a)

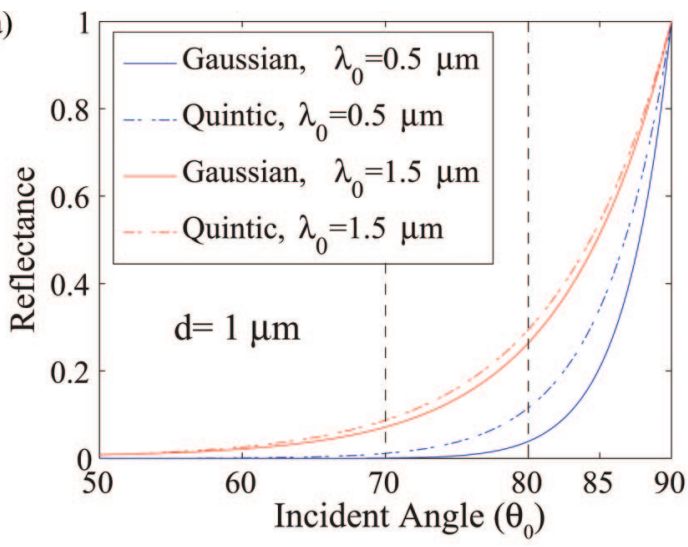

(b)

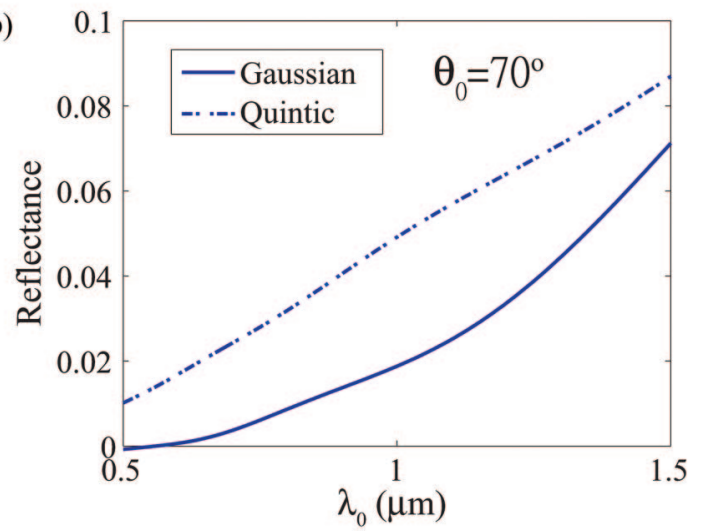

(c)

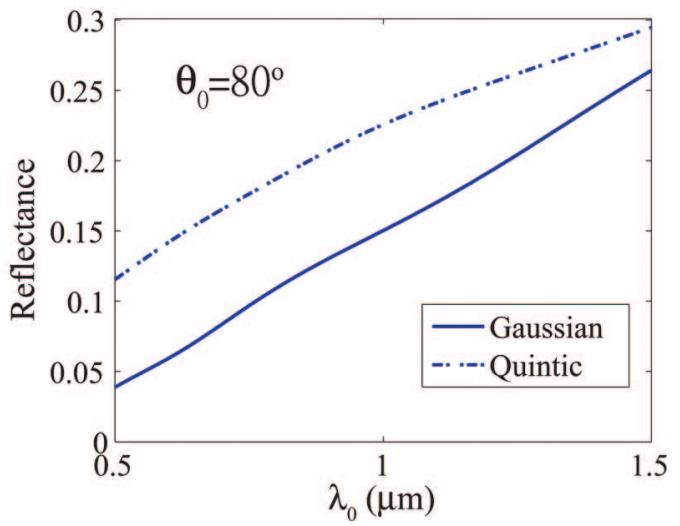

Fig. 4. (a) Gaussian and Quintic gradient-index coating angular reflectance calculated with a physical thickness of $1 \mu \mathrm{m}$. The spectral reflectance calculated with a physical thickness of $1 \mu \mathrm{m}$ for (b) $\theta_{0}=70^{\circ}$ incident angle and (c) $\theta_{0}=80^{\circ}$ incident angle.

TM polarizations. One can see the trend that the shorter wavelength performs better due to the relatively high ratio $\left(d / \lambda_{0}\right)$ of coating thickness to wavelength. We plot the reflectance as a function of wavelength at two angles of incidence, $\theta_{0}=70^{\circ}$ and $\theta_{0}=80^{\circ}$ in Fig. 4. Clearly, the Gaussian is better over a wide range of wavelength. It was previously shown that the Gaussian profile can have better performance than the Quintic at normal incidence [9]. It is shown here that the same holds true at oblique incidence, and we can consider the refractive angle within the two profiles to gain some physical understanding. (a)

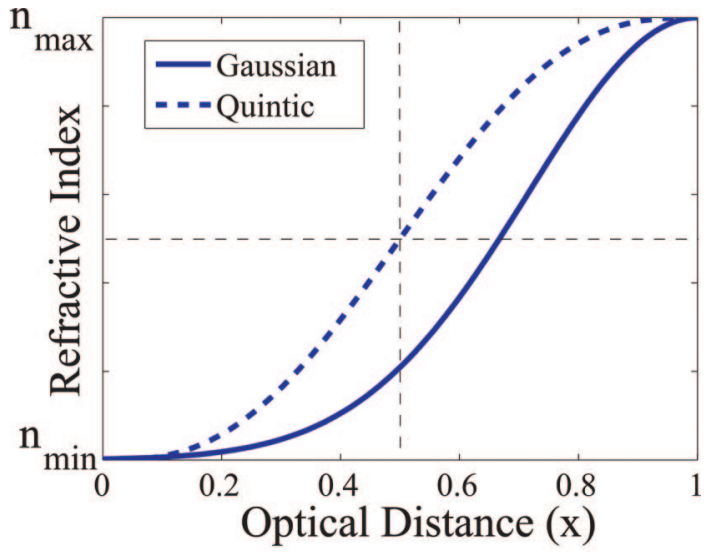

(b)

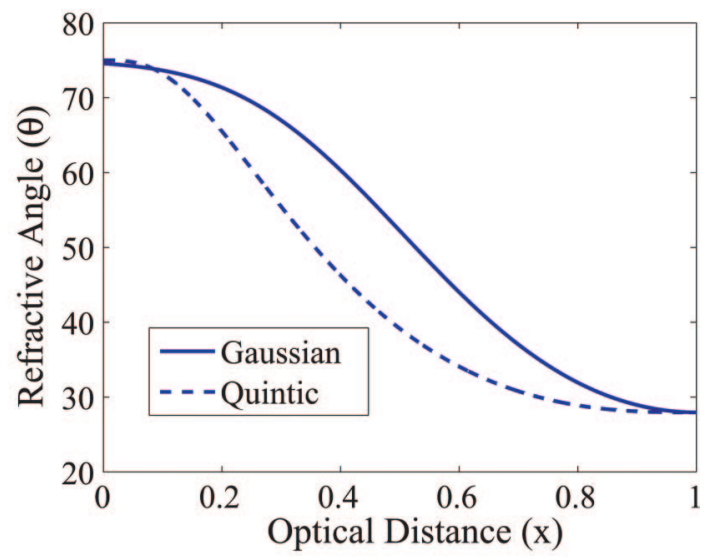

(c)

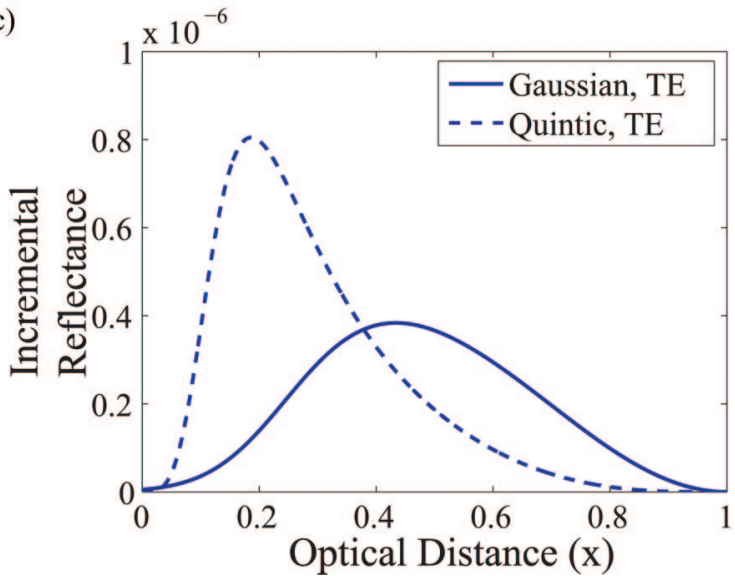

Fig. 5. (Color online) (a) Index profile plotted for the Gaussian (solid curve) and Quintic profile (dash curve), respectively. Those two profiles are normalized to optical distance. (b) Refractive angle within the Gaussian and Quintic coating. (c) Reflectance within the Gaussian and Quintic coating at incident angle of $\theta_{0}=75^{\circ}$.

In Fig. 5(a), we plot both Quintic and Gaussian profiles as a function of optical distance. Figure 5(b) shows the refractive angle within the Gaussian as well as Quintic profile as function of optical distance at $\theta=75^{\circ}$, and Fig. 5(c) gives the incremental reflectance as a function of optical distance for the two profiles. The refractive angle $\theta$ in Quintic has a dramatic change at the front region whereas that of the Gaussian is relatively uniformly varied over the 
whole coating. Consequently, Quintic gains its top reflectance at about $x=0.18$ as shown in Fig. 5(c) while Gaussian has its top reflectance relatively close to the central point. In a nonstrict sense, the integration of reflectance over the whole coating indicates the total reflectance from the inhomogeneous profile. Clearly, the reflectance under Quintic covers more area than that under Gaussian as revealed in Fig. 5(c). Therefore, Gaussian gives a better ARC performance than Quintic, which agrees with our assertion. At a given coating thickness, the smoothness of the refractive angle can thus be used as a criterion in the synthesis of optimal gradient-index profile for wideangle operation. Alternatively, given a pool of candidate profiles, the best one for large incident angles can be selected simply by comparing their respective refractive angle profiles.

\section{B. Discretization}

In the following, we consider the discretization of the continuous profiles [20-22] and how it affects the overall performance. It was recently, demonstrated that a large range of arbitrary effective indexes may be realized in a layer of nanorods grown by glacing angle deposition [22]. Therefore we do not put restrictions on the index of our discretized layers. The re-

(a)

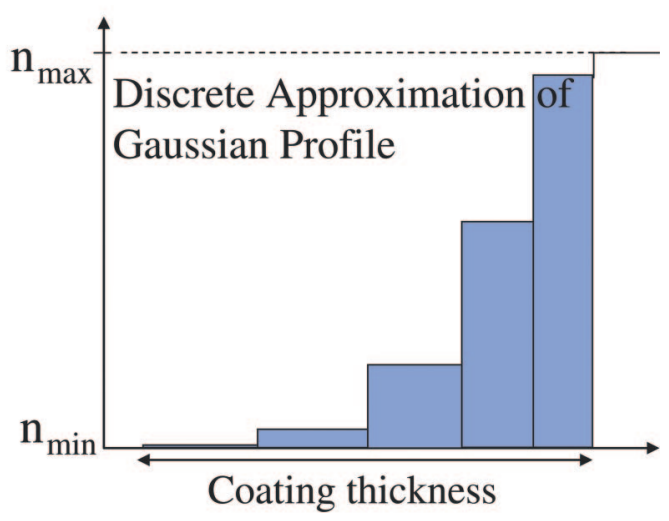

(b)

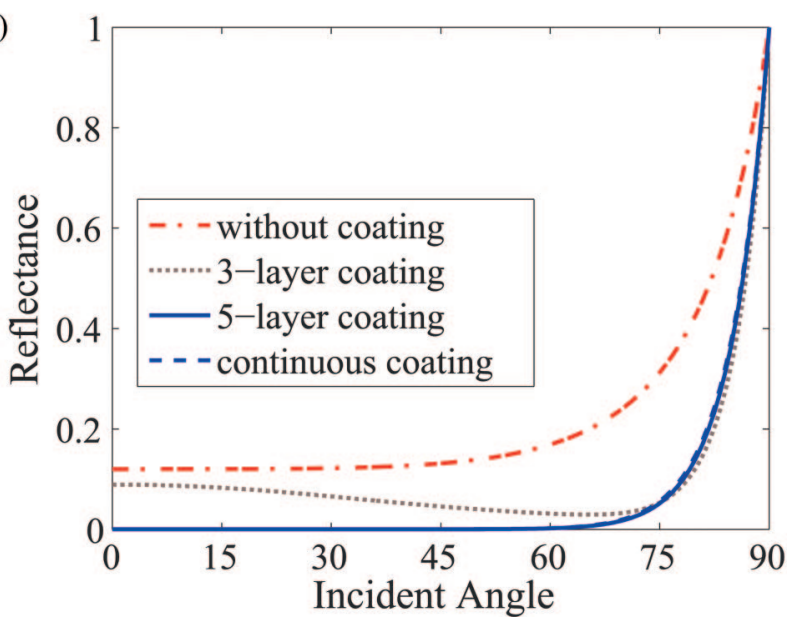

Fig. 6. (Color online) (a) Five-layer discretized Gaussian profile. (b) Angular reflectance with respect to different numbers of discrete layers for a Gaussian profile. fractive index and thickness of each layer are obtained by the following procedure:

- The refractive index is obtained by sampling the original profile at the center of a layer and with equal layer spacing $(\Delta x)$.

- The thickness of each layer is obtained by performing the integration of Eq. (1), with limits from $-x / 2$ to $x / 2$, centering at the sample point.

Figure 6(a) illustrates the geometry of ARC consisting of five laminar layers to approximate Gaussian profile. Note that the interference between both ends of each laminar layer is the same. Figure $6(\mathrm{~b})$ shows the calculated results with various numbers of layers. It can be seen that as few as five discrete homogeneous layers are sufficient for approximating Gaussian for ARC between air and AIN that show good performance. This is a direct consequence of the fact that the fine structure is not resolvable under one-fifth of a wavelength with an index variation from 1 to 2.06. If we increase the index variation, more than five layers may be necessary to approximate the original profile.

\section{Conclusion}

At large incident angles, the magnitude of total reflection from a gradient-index film is mainly influenced by the smoothness of the optical path inside the film. The smoothness of the optical path can thus be used as a design criterion for omnidirectional ARC profile. This point of view can also be used to explain the performance difference between gradient-index profiles such as the Gaussian and Quintic. In addition, the Gaussian profile can be sufficiently approximated by as little as five discrete homogeneous layers for an ARC between air and AIN.

The authors acknowledge partial financial support of this work from the U.S. Department of Energy, Sandia National Laboratory. M. Chen acknowledges the financial support of the National Science Council of Republic of China under the "Graduate Student Study Abroad Program” with grant 095-2917-I-002-026.

\section{References}

1. A. Mussett and A. Thelen, "Multilayer antireflection coatings," in Progress in Optics Vol. 8, E. Wolf, ed. (North-Holland, 1970), pp. 203-237.

2. J. A. Dobrowolski, "Optical properties of films and coatings," in Handbook of Optics, M. Bass, ed. (McGraw-Hill, 1995), pp. 42.19-42.34.

3. A. Thelen, Design of Optical Interference Coatings (McGrawHill, 1989).

4. R. Jacobsson, "Light reflection from films of continuously varying refractive index," in Progress in Optics, Vol. 5, E. Wolf, ed. (North-Holland, 1966), pp. 247-286.

5. W. H. Southwell, "Gradent-index antireflection coatings," Opt. Lett. 8, 584-586 (1983).

6. E. Spiller, I. Haller, R. Feder, J. E. E. Baglin, and W. N. Hammer, "Graded-index AR surfaces produce by ion implantation on plastic materials," Appl. Opt. 19, 3022-3026 (1980).

7. P. Yeh and S. Sari, "Optical properties of stratified media with 
exponentially graded refractive index," Appl. Opt. 22, 41424145 (1983).

8. P. G. Verly, J. A. Dobrowolski, and R. R. Willey, "Fouriertransform method for the design of wideband antirefretion coatings," Appl. Opt. 31, 3836-3846 (1992).

9. E. B. Grann, M. G. Moharam, and D. A. Pommet, "Optimal design for antireflective tapered two-dimensional subwavelength grating structures,” J. Opt. Soc. Am. A 12, 333-339 (1995).

10. M. J. Minot, "The angular reflectance of single-layer gradient refractive-index films," J. Opt. Soc. Am. 67, 1046-1050 (1977).

11. A. Gombert, W. Glaubitt, K. Rose, J. Dreibholz, C. Zanke, B. Blasi, A. Heinzel, W. Horbelt, D. Sporn, W. Doll, V. Wittwer, and J. Luther, "Grazing with very high solar transmittance," Sol. Energy 62, 177-188 (1998).

12. Y. Kanamori, M. Ishimori, and K. Hane, "High efficient lightemitting diodes with antireflection subwavelength gratings," IEEE Photon. Technol. Lett. 14, 1064-1066 (2002).

13. P. B. Clapham and M. C. Hutley, "Reduction of lens reflexion by the 'moth eye' principle," Nature 244, 281-282 (1973).

14. P. Baumeister, "The transmission and degree of polarization of quarter-wave stacks at nonnormal incidence," Opt. Acta. 8, 105-119 (1961).
15. J. A. Dobrowolski and S. H. C. Piotrowski, "Refractive index as a variable in the numerical design of optical thin films," Appl. Opt. 21, 1502-1510 (1982).

16. J. C. Monga, "Anti-reflection coatings for grazing incidence angles,” J. Mod. Opt. 36, 381-387 (1989).

17. J. C. Monga, "Double-layer broadband antireflection coatings for grazing incidence angles," Appl. Opt. 31, 546-553 (1992).

18. J. A. Dobrowolski, D. Poitras, P. Ma, H. Vakil, and M. Acree, "Toward perfect antireflection coatings: numerical investigation," Appl. Opt. 41, 3075-3083 (2002).

19. D. Poitras and J. A. Dobrowolski, "Toward perfect antireflection coatings 2: Theory," Appl. Opt. 43, 1286-1295 (2004).

20. W. H. Southwell, "Coating design using very thin high-andlow-index layers,” Appl. Opt. 24, 457-460 (1985).

21. H. Sankur and W. H. Southwell, "Broadband gradient-index antireflection coating for ZnSe," Appl. Opt. 23, 2770-2773 (1984).

22. J.-Q. Xi, J. K. Kim, E. F. Schubert, D. Ye, T. M. Lu, S. Y. Lin, and J. S. Juneja, "Very low-refractive-index optical thin films consisting of an array of $\mathrm{SiO}_{2}$ nanorods," Opt. Lett. 31, 601603 (2006). 